\title{
Triple Positive Solutions of a Nonlocal Boundary Value Problem for Singular Differential Equations with $p$-Laplacian
}

\author{
Jufang Wang, Changlong Yu, and Yanping Guo \\ College of Sciences, Hebei University of Science and Technology, Shijiazhuang, Hebei 050018, China \\ Correspondence should be addressed to Jufang Wang; wangjufang1981@126.com and Changlong Yu; changlongyu@126.com
}

Received 22 October 2012; Revised 13 January 2013; Accepted 26 January 2013

Academic Editor: Bashir Ahmad

Copyright (C) 2013 Jufang Wang et al. This is an open access article distributed under the Creative Commons Attribution License, which permits unrestricted use, distribution, and reproduction in any medium, provided the original work is properly cited.

We establish the existence of triple positive solutions of an $m$-point boundary value problem for the nonlinear singular secondorder differential equations of mixed type with a $p$-Laplacian operator by Leggett-William fixed point theorem. At last, we give an example to demonstrate the use of the main result of this paper. The conclusions in this paper essentially extend and improve the known results.

\section{Introduction}

The existence and multiplicity of positive solutions for differential equations boundary value problems (BVPs) with the $p$ Laplacian operator subject to Dirichlet, Sturm-Liouville, or nonlinear boundary value conditions have been extensively investigated in recent years; see [1-10] and the references therein. Particularly, the following differential equations with one-dimensional $p$-Laplacian

$$
\left(\phi_{p}\left(u^{\prime}\right)\right)^{\prime}+q(t) f(t, u)=0, \quad 0 \leq t \leq 1
$$

have been studied subject to different kinds of boundary conditions; see [1-4] and the references therein. The methods mainly depend on Kransnosel'skii fixed point theorem, upper and lower solution technique, Leggett-Williams fixed point theorem, and some new fixed point theorems in cones, and so forth.

Recently, in [9], Kong et al. have studied the existence of triple positive solutions for the following BVP:

$$
\begin{aligned}
& \left(\phi_{p}\left(u^{\prime}\right)\right)^{\prime}(t)+q(t) f \\
& \times\left(u(t), u^{\prime}(t),(T u)(t),(S u)(t)\right)=0, \quad 0 \leq t \leq 1, \\
& u^{\prime}(0)=0, \quad u(1)=g\left(u^{\prime}(1)\right) .
\end{aligned}
$$

More recently, in [10], $\mathrm{Hu}$ and $\mathrm{Ma}$ have pointed out that the equivalent integral equation of BVP (2) is wrong in [9] and studied the existence of triple positive solutions for the following BVP:

$$
\begin{aligned}
& \left(\phi_{p}\left(u^{\prime}\right)\right)^{\prime}(t)+q(t) f \\
& \times\left(u(t), u^{\prime}(t),(T u)(t),(S u)(t)\right)=0, \quad 0 \leq t \leq 1, \\
& u^{\prime}(0)=\beta u^{\prime}(\eta), \quad u(1)=g\left(u^{\prime}(1)\right) .
\end{aligned}
$$

Firstly, we confirm that the mistakes which have been pointed out in [10] exist. At the same time, we think that the value of $M$ designed in Theorem 3.1 in [10] is not suitable, since the proof needs the condition $\phi_{q}(M) \leq M$, but in fact this condition does not always hold.

Motivated by the work above, in this paper, we will study the following more extensive second-order $m$-point BVP:

$$
\begin{aligned}
& \left(\phi_{p}\left(u^{\prime}\right)\right)^{\prime}(t)+q(t) f \\
& \quad \times\left(u(t), u^{\prime}(t),(T u)(t),(S u)(t)\right)=0, \quad 0 \leq t \leq 1, \\
& \phi_{p}\left(u^{\prime}(0)\right)=\sum_{i=1}^{m-2} \beta_{i} \phi_{p}\left(u^{\prime}\left(\eta_{i}\right)\right), \quad u(1)=g\left(u^{\prime}(1)\right),
\end{aligned}
$$


where $\phi_{p}(s)=|s|^{p-2} s(p>1)$ is an increasing function, $\left(\phi_{p}\right)^{-1}(s)=\phi_{q}(s), 1 / p+1 / q=1 ; 0 \leq \beta_{i}<1, i=1,2, \ldots$, $m-2, \sum_{i=1}^{m-2} \beta_{i}<1$, and $0<\eta_{1}<\eta_{2}<\cdots<\eta_{m-2}$; $T$ and $S$ are two linear operators defined by

$$
\begin{array}{r}
T u(t)=\int_{0}^{t} k(t, s) u(s) d s, \\
S u(t)=\int_{0}^{1} h(t, s) u(s) d s, \\
u \in C^{1}[0,1],
\end{array}
$$

in which $k \in C\left[D, R^{+}\right], h \in C\left[D_{0}, R^{+}\right], D=\left\{(t, s) \in R^{2}\right.$ : $0 \leq s \leq t \leq 1\}, D_{0}=\left\{(t, s) \in R^{2}: 0 \leq s, t \leq 1\right\}, R^{+}=$ $[0,+\infty), R=(-\infty,+\infty), k_{0}=\max \{k(t, s):(t, s) \in D\}$, and $h_{0}=\max \left\{h(t, s):(t, s) \in D_{0}\right\}$.

Obviously, when $m=3$, BVP (4) reduces to BVP (3), and when $m=3, \beta_{1}=0, \operatorname{BVP}(4)$ reduces to $\operatorname{BVP}(2)$, so BVP (2) and BVP (3) are special cases of BVPs (4).

Throughout this paper, we always suppose the following conditions hold:

$\left(\mathrm{C}_{1}\right) f \in C\left(R^{+} \times R \times R^{+} \times R^{+},(0,+\infty)\right)$;

$\left(\mathrm{C}_{2}\right) q(t) \in C\left([0,1], R^{+}\right)$may be singular at $t=0,1$ and $0<$ $\int_{0}^{1} q(t) d t<+\infty$, so it is easy to see that there exists a constant $M>0$ such that $0<\int_{0}^{1} q(t) d t<\phi_{p}(M)$;

$\left(\mathrm{C}_{3}\right) g: R \rightarrow R^{+}$is nonincreasing and continuous, and $0 \leq g(v) \leq|v|$ for $v \in R$.

\section{Preliminary Results}

In this section, we firstly present some definitions, theorems, and lemmas, which will be needed in the proof of the main result.

Definition 1. Let $E$ be a real Banach space. A nonempty closed convex set $P \subset E$ is called a cone if it satisfies the following two conditions:

(i) $x \in P, \lambda \geq 0$ implies $\lambda x \in P$;

(ii) $x \in P,-x \in P$ implies $x=0$.

Definition 2. Given a cone $P$ in a real Banach space $E$, a continuous map $\alpha$ is called a concave (resp., convex) functional on $P$ if and only if, for all $x, y \in P$ and $0 \leq t \leq 1$, it holds:

$$
\begin{aligned}
& \alpha(t x+(1-t) y) \geq t \alpha(x)+(1-t) \alpha(y), \\
& (\operatorname{resp} ., \alpha(t x+(1-t) y) \leq t \alpha(x)+(1-t) \alpha(y)) .
\end{aligned}
$$

We consider the Banach space $E=C^{1}[0,1]$ equipped with norm $\|u\|=\max _{0 \leq t \leq 1}\left\{\|u(t)\|_{0},\left\|u^{\prime}(t)\right\|_{0}\right\}$, where $\|u\|_{0}=$ $\max _{0 \leq t \leq 1}|u(t)|$.

We denote, for any fixed constants $a, b, r$,

$C^{+}[0,1]=\{u \in C[0,1]: u(t) \geq 0, t \in[0,1]\}$,

$P=\{u \in E \mid u(t)$ is concave and nonincreasing on $[0,1]\}$,

$$
\begin{aligned}
& P_{r}=\{u \in P:\|u\|<r\}, \\
& P(\alpha, a, b)=\{u \in P: a \leq \alpha(u),\|u\|<b\} .
\end{aligned}
$$

It's easy to see that $P$ is a cone in $E$.

Theorem 3 (Leggett-William). Let $A: \bar{P}_{c} \rightarrow \bar{P}_{c}$ be a completely continuous map and let $\alpha$ be a nonnegative continuous concave functional on $P$ with $\alpha(u) \leq\|u\|$ for any $u \in \bar{P}_{c}$. Suppose there exist constants $a, b$, and $d$ with $0<a<$ $b<d \leq c$ such that

(i) $\{u \in P(\alpha, b, d): \alpha(u)>b\} \neq \phi$ and $\alpha(A u)>b$ for all $u \in P(\alpha, b, d)$;

(ii) $\|A u\|<$ a for all $u \in \bar{P}_{a}$;

(iii) $\alpha(A u)>b$ for all $u \in P(\alpha, b, c)$ with $\|A u\|>d$.

Then $A$ has at least three fixed points $u_{1}, u_{2}$, and $u_{3}$ satisfying

$$
\begin{array}{cc}
\left\|u_{1}\right\|<a, & b<\alpha\left(u_{2}\right), \\
\left\|u_{3}\right\|>a, & \alpha\left(u_{3}\right)<b .
\end{array}
$$

Lemma 4. Suppose $y \in C^{1}[0,1]$ with $\left(\phi_{p}\left(y^{\prime}\right)\right)^{\prime} \in L^{1}[0,1]$ satisfies

$$
\begin{gathered}
\left(\phi_{p}\left(y^{\prime}\right)\right)^{\prime}(t) \leq 0, \quad 0 \leq t \leq 1, \\
\phi_{p}\left(y^{\prime}(0)\right)=\sum_{i=1}^{m-2} \beta_{i} \phi_{p}\left(y^{\prime}\left(\eta_{i}\right)\right), \quad y(1)=g\left(y^{\prime}(1)\right) .
\end{gathered}
$$

Then, $y(t) \geq 0$ is concave and nonincreasing on $[0,1]$, that is, $y \in P$.

Proof. Since $\left(\phi_{p}\left(y^{\prime}\right)\right)^{\prime}(t) \leq 0$, we know that $\phi_{p}\left(y^{\prime}\right)$ is nonincreasing, that is, $y^{\prime}(t)$ is nonincreasing, which means $y(t)$ is concave. At the same time, we have $y^{\prime}(t) \leq y^{\prime}(0)$, so $y^{\prime}(0)=\phi_{q}\left(\sum_{i=1}^{m-2} \beta_{i} \phi_{p}\left(y^{\prime}\left(\eta_{i}\right)\right)\right) \leq \phi_{q}\left(\sum_{i=1}^{m-2} \beta_{i} \phi_{p}\left(y^{\prime}(0)\right)\right)=$ $\phi_{q}\left(\sum_{i=1}^{m-2} \beta_{i}\right) y^{\prime}(0)$, namely $y^{\prime}(0) \leq 0$. Then, $y^{\prime}(t) \leq 0$; that is to say, $y(t)$ is nonincreasing. So $y(t) \geq y(1)=g\left(y^{\prime}(1)\right) \geq 0$. Above all, $y \in P$. This completes the proof.

Lemma 5. Let $y \in C[0,1]$ and $\left(\phi_{p}\left(u^{\prime}\right)\right)^{\prime} \in L^{1}[0,1]$, then, $B V P$

$$
\begin{gathered}
\left(\phi_{p}\left(u^{\prime}\right)\right)^{\prime}(t)=-y(t), \quad 0 \leq t \leq 1, \\
\phi_{p}\left(u^{\prime}(0)\right)=\sum_{i=1}^{m-2} \beta_{i} \phi_{p}\left(u^{\prime}\left(\eta_{i}\right)\right), \quad u(1)=g\left(u^{\prime}(1)\right),
\end{gathered}
$$

has a unique solution

$$
\begin{aligned}
u(t)= & \int_{t}^{1} \phi_{q}\left(\frac{1}{1-\beta} \sum_{i=1}^{m-2} \beta_{i} \int_{0}^{\eta_{i}} y(r) d r+\int_{0}^{s} y(r) d r\right) d s \\
& +g\left(-\phi_{q}\left(\frac{1}{1-\beta} \sum_{i=1}^{m-2} \beta_{i} \int_{0}^{\eta_{i}} y(r) d r+\int_{0}^{1} y(r) d r\right)\right),
\end{aligned}
$$

where $\beta=\sum_{i=1}^{m-2} \beta_{i}$. 
Define the operator $A: P \rightarrow E$ by

$$
\begin{array}{r}
(A u)(t)=\int_{t}^{1} \phi_{q}\left(\frac{1}{1-\beta} \sum_{i=1}^{m-2} \beta_{i} \int_{0}^{\eta_{i}} q(r) f\left(u, u^{\prime}, T u, S u\right) d r\right. \\
\left.\quad+\int_{0}^{s} q(r) f\left(u, u^{\prime}, T u, S u\right) d r\right) d s \\
+g\left(-\phi_{q}\left(\frac{1}{1-\beta} \sum_{i=1}^{m-2} \beta_{i} \int_{0}^{\eta_{i}} q(r)\right.\right. \\
\times f\left(u, u^{\prime}, T u, S u\right) d r \\
\left.\left.+\int_{0}^{1} q(r) f\left(u, u^{\prime}, T u, S u\right) d r\right)\right) .
\end{array}
$$

Obviously, $A$ is well defined and $u \in E$ is a solution of BVP (4) if and only if $u$ is a fixed point of $A$.

Lemma 6. $A: P \rightarrow P$ is completely continuous.

Proof. It is similar to the proof of Lemma 2.2 in [9].

Lemma 7. For any $u \in P$, one has $\|A u\|_{0} \leq 2\left\|(A u)^{\prime}\right\|_{0},\|A u\| \leq$ $2\left\|(A u)^{\prime}\right\|_{0}$.

Proof. From (10), we obtain

$$
\begin{gathered}
\|(A u)\|_{0}=\int_{0}^{1} \phi_{q}\left(\frac{1}{1-\beta} \sum_{i=1}^{m-2} \beta_{i} \int_{0}^{\eta_{i}} q(r) f\left(u, u^{\prime}, T u, S u\right) d r\right. \\
\left.+\int_{0}^{s} q(r) f\left(u, u^{\prime}, T u, S u\right) d r\right) d s \\
+g\left(-\phi_{q}\left(\frac{1}{1-\beta} \sum_{i=1}^{m-2} \beta_{i} \int_{0}^{\eta_{i}} q(r)\right.\right. \\
\times f\left(u, u^{\prime}, T u, S u\right) d r \\
\leq \int_{0}^{1} \phi_{q}\left(\frac{1}{1-\beta} \sum_{i=1}^{m-2} \beta_{i} \int_{0}^{\eta_{i}} q(r) f\left(u, u^{\prime}, T u, S u\right) d r\right. \\
\left.+\int_{0}^{s} q(r) f\left(u, u^{\prime}, T u, S u\right) d r\right) d s
\end{gathered}
$$

$$
\begin{aligned}
& +\phi_{q}\left(\frac{1}{1-\beta} \sum_{i=1}^{m-2} \beta_{i} \int_{0}^{\eta_{i}} q(r) f\left(u, u^{\prime}, T u, S u\right) d r\right. \\
& \left.\quad+\int_{0}^{1} q(r) f\left(u, u^{\prime}, T u, S u\right) d r\right) \\
& =2 \phi_{q}\left(\frac{1}{1-\beta} \sum_{i=1}^{m-2} \beta_{i} \int_{0}^{\eta_{i}} q(r) f\left(u, u^{\prime}, T u, S u\right) d r\right. \\
& \left.\quad+\int_{0}^{1} q(r) f\left(u, u^{\prime}, T u, S u\right) d r\right) \\
& =2\left|(A u)^{\prime}(1)\right| \\
& =2\left\|(A u)^{\prime}\right\|_{0} .
\end{aligned}
$$

Since $\|A u\|=\max \left\{\|A u\|_{0},\left\|(A u)^{\prime}\right\|_{0}\right\}$, so we have $\|A u\| \leq$ $2\left\|(A u)^{\prime}\right\|_{0}$, which completes the proof.

\section{Main Results}

For any $\delta \in\left(0, \min \left\{\eta_{1}, 1 / 2\right\}\right)$, we define a nonnegative continuous concave function $\alpha: P \rightarrow R^{+}$by $\alpha(u)=$ $\min _{\delta \leq t \leq(1-\delta)} u(t)$. Obviously, the following two conclusions hold:

$$
\begin{array}{r}
\alpha(u)=u(1-\delta) \leq\|u\|_{0}, \quad \alpha(A u)=A u(1-\delta), \\
\forall u \in P .
\end{array}
$$

The main result of this paper is following.

Theorem 8. Let $m_{0}=\min _{0 \leq t \leq 1} q(t)$ and $\beta=\sum_{i=1}^{m-2} \beta_{i}<1$. Suppose $\left(C_{1}\right),\left(C_{2}\right)$, and $\left(C_{3}\right)$ hold. Suppose further that there exist numbers $\delta \in\left(0, \min \left\{\eta_{1}, 1 / 2\right\}\right), a, b, c$, and $d$ such that $0<a<b \leq m_{0} \delta d / M<d \leq c$, and

$\left(\mathrm{H}_{1}\right) f(u, v, w, l) \leq(1-\beta) \phi_{p}(a / 2 M)$, for $(u, v, w, l) \in$ $[0, a] \times[-a, 0] \times\left[0, k_{0} a\right] \times\left[0, h_{0} a\right] ;$

$\left(\mathrm{H}_{2}\right) f(u, v, w, l) \leq(1-\beta) \phi_{p}(c / 2 M)$, for $(u, v, w, l) \in$ $[0, c] \times[-c, 0] \times\left[0, k_{0} c\right] \times\left[0, h_{0} c\right] ;$

$\left(\mathrm{H}_{3}\right) f(u, v, w, l)>\phi_{p}(b / \delta L)$, for $(u, v, w, l) \in[b, d] \times$ $[-d, 0] \times\left[0, k_{0} d\right] \times\left[0, h_{0} d\right]$, where $L=\phi_{q}\left(\int_{0}^{1-\delta} q(t) d t\right) ;$

$\left(\mathrm{H}_{4}\right) \min _{(u, v, w, l) \in J} f(u, v, w, l) \phi_{p}\left(M /\left(2 m_{0}\right)\right) \int_{0}^{1-\delta} q(t) d t \geq$ $\max _{(u, v, w, l) \in J} f(u, v, w, l) \int_{0}^{1} q(t) d t$, where $J=[0, c] \times$ $[-c, 0] \times\left[0, k_{0} c\right] \times\left[0, h_{0} c\right]$.

Then, BVP (4) has at least three positive solutions $u_{1}, u_{2}$, and $u_{3}$ such that

$$
\begin{aligned}
& \left\|u_{1}\right\|<a, \quad b<\min _{\delta \leq t<(1-\delta)} u_{2}(t), \\
& \left\|u_{3}\right\|>a, \quad \min _{\delta \leq t<(1-\delta)} u_{3}(t)<b .
\end{aligned}
$$


Proof. We divide the proof into three steps.

Step 1. We prove $A \bar{P}_{c} \subset \bar{P}_{c}, A \bar{P}_{a} \subset \bar{P}_{a}$; that is, (ii) of Theorem 3. By Lemma 6, we have $A \bar{P}_{c} \subset P$, so $\forall u \in \bar{P}_{c}$, we get $0 \leq u(t) \leq c,-c \leq u^{\prime}(t) \leq 0,0 \leq(T u)(t) \leq k_{0} c$, $0 \leq(\mathrm{Su})(t) \leq h_{0} c$. For $t \in[0,1]$ and by $\left(\mathrm{H}_{2}\right)$

$\|(A u)\|_{0}$

$$
\begin{gathered}
=\int_{0}^{1} \phi_{q}\left(\frac{1}{1-\beta} \sum_{i=1}^{m-2} \beta_{i} \int_{0}^{\eta_{i}} q(r)\right. \\
\times f\left(u, u^{\prime}, T u, S u\right) d r \\
\left.+\int_{0}^{s} q(r) f\left(u, u^{\prime}, T u, S u\right) d r\right) d s \\
+g\left(-\phi_{q}\left(\frac{1}{1-\beta} \sum_{i=1}^{m-2} \beta_{i} \int_{0}^{\eta_{i}} q(r)\right.\right. \\
\times f\left(u, u^{\prime}, T u, S u\right) d r \\
\left.\left.+\int_{0}^{1} q(r) f\left(u, u^{\prime}, T u, S u\right) d r\right)\right)
\end{gathered}
$$$$
\leq \int_{0}^{1} \phi_{q}\left(\frac{1}{1-\beta} \sum_{i=1}^{m-2} \beta_{i} \int_{0}^{\eta_{i}} q(r) f\left(u, u^{\prime}, T u, S u\right) d r\right.
$$$$
\left.+\int_{0}^{s} q(r) f\left(u, u^{\prime}, T u, S u\right) d r\right) d s
$$$$
+\phi_{q}\left(\frac{1}{1-\beta} \sum_{i=1}^{m-2} \beta_{i} \int_{0}^{\eta_{i}} q(r) f\left(u, u^{\prime}, T u, S u\right) d r\right.
$$$$
\left.+\int_{0}^{1} q(r) f\left(u, u^{\prime}, T u, S u\right) d r\right)
$$$$
=2 \phi_{q}\left(\frac{1}{1-\beta} \sum_{i=1}^{m-2} \beta_{i} \int_{0}^{\eta_{i}} q(r) f\left(u, u^{\prime}, T u, S u\right) d r\right.
$$$$
\left.+\int_{0}^{1} q(r) f\left(u, u^{\prime}, T u, S u\right) d r\right)
$$$$
\leq 2 \phi_{q}\left(\frac{1}{1-\beta} \int_{0}^{1} q(r) f\left(u, u^{\prime}, T u, S u\right) d r\right)
$$$$
\leq 2 \phi_{q}\left(\phi_{p}\left(\frac{c}{2 M}\right)\right) \phi_{q}\left(\int_{0}^{1} q(r) d r\right)
$$$$
\leq c \text {. }
$$

$$
\begin{aligned}
\left\|(A u)^{\prime}\right\|_{0}=\phi_{q}( & \frac{1}{1-\beta} \sum_{i=1}^{m-2} \beta_{i} \int_{0}^{\eta_{i}} q(r) f\left(u, u^{\prime}, T u, S u\right) d r \\
& \left.+\int_{0}^{s} q(r) f\left(u, u^{\prime}, T u, S u\right) d r\right)
\end{aligned}
$$

$$
\begin{aligned}
& \leq \phi_{q}\left(\frac{1}{1-\beta} \sum_{i=1}^{m-2} \beta_{i} \int_{0}^{\eta_{i}} q(r) f\left(u, u^{\prime}, T u, S u\right) d r\right. \\
& \left.\quad+\int_{0}^{1} q(r) f\left(u, u^{\prime}, T u, S u\right) d r\right) \\
& \leq \phi_{q}\left(\frac{1}{1-\beta} \int_{0}^{1} q(r) f\left(u, u^{\prime}, T u, S u\right) d r\right) \\
& \leq \phi_{q}\left(\phi_{p}\left(\frac{c}{2 M}\right)\right) \phi_{q}\left(\int_{0}^{1} q(r) d r\right) \\
& \leq c .
\end{aligned}
$$

Hence, $\|A u\|<c$ and $A \bar{P}_{c} \subset \bar{P}_{c}$. Similarly, we obtain $A \bar{P}_{a} \subset$ $\bar{P}_{a}$.

Step 2. We show

$$
\begin{aligned}
& \{u \in P(\alpha, b, d): \alpha(u)>b\} \neq \phi, \\
& \alpha(A u)>b, \quad \forall u \in P(\alpha, b, d),
\end{aligned}
$$

that is, (i) of Theorem 3.

Let $u=(b+d) / 2$, then $u \in P(\alpha, b, d), \alpha(u)=(b+$ $d) / 2>b$. Hence, (15) holds. For any $u \in P(\alpha, b, d)$, we have $b \leq u(t) \leq d,-d \leq u^{\prime}(t) \leq 0,0 \leq(T u)(t) \leq k_{0} d, 0 \leq$ $(\mathrm{Su})(t) \leq h_{0} d, t \in[0,1-\delta]$, so by $\left(\mathrm{H}_{3}\right)$, we have

$$
\begin{aligned}
& \alpha(A u)= \min _{t \in[\delta, 1-\delta]}(A u)(t)=(A u)(1-\delta) \\
&=\int_{1-\delta}^{1} \phi_{q}\left(\frac{1}{1-\beta} \sum_{i=1}^{m-2} \beta_{i} \int_{0}^{\eta_{i}} q(r) f\left(u, u^{\prime}, T u, S u\right) d r\right. \\
&\left.+\int_{0}^{s} q(r) f\left(u, u^{\prime}, T u, S u\right) d r\right) d s \\
&+g\left(-\phi_{q}\left(\frac{1}{1-\beta} \sum_{i=1}^{m-2} \beta_{i} \int_{0}^{\eta_{i}} q(r) f\left(u, u^{\prime}, T u, S u\right) d r\right.\right. \\
& \geq \delta \phi_{q}\left(\frac{1}{1-\beta} \sum_{i=1}^{m-2} \beta_{i} \int_{0}^{\eta_{i}} q(r) f\left(u, u^{\prime}, T u, S u\right) d r\right. \\
&\left.+\int_{0}^{1-\delta} q(r) f\left(u, u^{\prime}, T u, S u\right) d r\right) \\
&+g\left(-\phi_{q}\left(\frac{1}{1-\beta} \sum_{i=1}^{m-2} \beta_{i} \int_{0}^{\eta_{i}} q(r) f\left(u, u^{\prime}, T u, S u\right) d r\right.\right. \\
&\left.\left.+\int_{0}^{1} q(r) f\left(u, u^{\prime}, T u, S u\right) d r\right)\right)
\end{aligned}
$$




$$
\begin{aligned}
& \geq \delta \phi_{q}\left(\int_{0}^{1-\delta} q(r) f\left(u, u^{\prime}, T u, S u\right) d r\right) \\
& \geq \delta \phi_{q}\left(\phi_{p}\left(\frac{b}{\delta L}\right)\right) \phi_{q}\left(\int_{0}^{1-\delta} q(r) d r\right) \\
& =b .
\end{aligned}
$$

Hence (16) holds.

Step 3. We show that $\alpha(A u)>b$ for all $u \in P(\alpha, b, c)$ with $\|A u\|>d$, that is, (iii) of Theorem 3 .

If $u \in P(\alpha, b, c)$ with $\|A u\|>d$, we obtain $0 \leq u(t) \leq$ $c,-c \leq u^{\prime}(t) \leq 0,0 \leq(T u)(t) \leq k_{0} c, 0 \leq(S u)(t) \leq h_{0} c$, for any $t \in[0,1]$, and so by $\left(\mathrm{H}_{4}\right)$, we have

$$
\begin{gathered}
\phi_{p}\left(\frac{M}{2 m_{0}}\right) \int_{0}^{1-\delta} q(r) f\left(u, u^{\prime}, T u, S u\right) d r \\
\geq \int_{0}^{1} q(r) f\left(u, u^{\prime}, T u, S u\right) d r .
\end{gathered}
$$

Furthermore, we have

$$
\begin{gathered}
\phi_{p}\left(\frac{M}{2 m_{0}}\right) \int_{0}^{1-\delta} q(r) f\left(u, u^{\prime}, T u, S u\right) d r \\
+\phi_{p}\left(\frac{M}{2 m_{0}}\right) \frac{1}{1-\beta} \\
\quad \times \sum_{i=1}^{m-2} \beta_{i} \int_{0}^{\eta_{i}} q(r) f\left(u, u^{\prime}, T u, S u\right) d r \\
\geq \int_{0}^{1} q(r) f\left(u, u^{\prime}, T u, S u\right) d r \\
+\frac{1}{1-\beta} \sum_{i=1}^{m-2} \beta_{i} \int_{0}^{\eta_{i}} q(r) f\left(u, u^{\prime}, T u, S u\right) d r .
\end{gathered}
$$

Therefore, by Lemma 7, we have

$$
\begin{gathered}
\alpha(A u)=(A u)(1-\delta) \\
=\int_{1-\delta}^{1} \phi_{q}\left(\frac{1}{1-\beta} \sum_{i=1}^{m-2} \beta_{i} \int_{0}^{\eta_{i}} q(r)\right. \\
\quad \times f\left(u, u^{\prime}, T u, S u\right) d r \\
\left.+\int_{0}^{s} q(r) f\left(u, u^{\prime}, T u, S u\right) d r\right) d s \\
+g\left(-\phi_{q}\left(\frac{1}{1-\beta} \sum_{i=1}^{m-2} \beta_{i} \int_{0}^{\eta_{i}} q(r)\right.\right. \\
\left.\left.\quad+\int_{0}^{1} q(r) f\left(u, u^{\prime}, T u, S u\right) d r\right)\right)
\end{gathered}
$$

$$
\begin{gathered}
\geq \delta \phi_{q}\left(\frac{1}{1-\beta} \sum_{i=1}^{m-2} \beta_{i} \int_{0}^{\eta_{i}} q(r) f\left(u, u^{\prime}, T u, S u\right) d r\right. \\
\left.+\int_{0}^{1-\delta} q(r) f\left(u, u^{\prime}, T u, S u\right) d r\right)
\end{gathered}
$$$$
\geq \delta \phi_{q}\left(\left(\frac{1}{1-\beta} \sum_{i=1}^{m-2} \beta_{i} \int_{0}^{\eta_{i}} q(r)\right.\right.
$$$$
\times f\left(u, u^{\prime}, T u, S u\right) d r
$$$$
\left.+\int_{0}^{1} q(r) f\left(u, u^{\prime}, T u, S u\right) d r\right)
$$$$
\left.\times\left(\phi_{p}\left(\frac{M}{2 m_{0}}\right)\right)^{-1}\right)
$$$$
=\frac{2 \delta m_{0}}{M} \phi_{q}\left(\frac{1}{1-\beta} \sum_{i=1}^{m-2} \beta_{i} \int_{0}^{\eta_{i}} q(r)\right.
$$$$
\times f\left(u, u^{\prime}, T u, S u\right) d r
$$$$
\left.+\int_{0}^{1} q(r) f\left(u, u^{\prime}, T u, S u\right) d r\right)
$$$$
=\frac{2 \delta m_{0}}{M}\left|(A u)^{\prime}(1)\right|
$$$$
=\frac{2 \delta m_{0}}{M}\left\|(A u)^{\prime}\right\|_{0}
$$$$
\geq \frac{\delta m_{0}}{M}\left\|(A u)^{\prime}\right\|
$$$$
>\frac{\delta m_{0}}{M} d
$$$$
\geq b \text {. }
$$

Hence, by Theorem 3, the results of Theorem 8 hold. This completes the proof of Theorem 8 .

\section{Example}

Consider the following BVP:

$$
\begin{aligned}
\left(\left|u^{\prime}\right| u^{\prime}\right)^{\prime}(t)+q(t) & \\
\times f\left(u(t), u^{\prime}(t),(T u)(t),(S u)(t)\right) & =0, \\
0 \leq t \leq 1, &
\end{aligned}
$$




$$
\begin{aligned}
\left|u^{\prime}(0)\right| u^{\prime}(0)= & \frac{1}{4}\left|u^{\prime}\left(\frac{2}{5}\right)\right| u^{\prime}\left(\frac{2}{5}\right) \\
+ & \frac{1}{4}\left|u^{\prime}\left(\frac{1}{2}\right)\right| u^{\prime}\left(\frac{1}{2}\right), \\
u(1) & =g\left(u^{\prime}(1)\right),
\end{aligned}
$$

where

$$
\begin{gathered}
q(t)= \begin{cases}t^{-1 / 2}, & 0 \leq t \leq \frac{16}{25} \\
-\frac{187}{54} t+\frac{9359}{2700}, & \frac{16}{25} \leq t \leq 1 .\end{cases} \\
f(u, v, w, l)=\left\{\begin{aligned}
10 u^{10}+\frac{2+\sin v}{1000}+\frac{\sqrt[3]{w}}{1000}+\frac{\sqrt{l}}{1000} \\
0 \leq u \leq 1, v \leq 0, w, l \geq 0
\end{aligned}\right. \\
10 \sqrt[10]{u}+\frac{2+\sin v}{1000}+\frac{\sqrt[3]{w}}{1000}+\frac{\sqrt{l}}{1000} \\
g(v)= \begin{cases}v^{1 / 2}, & |v| \geq 1 \\
v^{2}, & |v|<1\end{cases}
\end{gathered}
$$

Proof. Since $M=\sqrt{2}$ and $m_{0}=1 / 300, \beta=1 / 2, \delta=$ $9 / 25, a=1 / 2, b=1, d=1296, c=1600, k(t, s)=1$ and $h(t, s)=1$, then we can obtain $0<a<b \leq\left(m_{0} \delta d\right) / M<d \leq$ $c$, and

$$
\begin{gathered}
L=\sqrt{\int_{0}^{1-9 / 25} q(t) d t}=\sqrt{\int_{0}^{16 / 25} t^{-1 / 2} d t}=\sqrt{\frac{8}{5}} \\
\phi_{p}\left(\frac{a}{2 M}\right)=\phi_{p}\left(\frac{1 / 2}{2 \sqrt{2}}\right)=\frac{1}{32} \\
\phi_{p}\left(\frac{c}{2 M}\right)=\phi_{p}\left(\frac{1600}{2 \sqrt{2}}\right)=320000 \\
\phi_{p}\left(\frac{b}{\delta L}\right)=\phi_{p}\left(\frac{1}{(9 / 25) \sqrt{8 / 5}}\right)=\frac{25^{2}}{9^{2}} \frac{5}{8}=\frac{3125}{648} .
\end{gathered}
$$

Next, we show that $\left(\mathrm{H}_{1}\right)-\left(\mathrm{H}_{4}\right)$ are satisfied.

If $0 \leq u \leq 1 / 2,-1 / 2 \leq v \leq 0,0 \leq w, l \leq 1 / 2$, then

$$
\begin{aligned}
f(u, v, w, l) & <10 \frac{1}{2}^{10}+\frac{3}{1000}+\frac{2}{1000} \\
& <(1-\beta) \phi_{p}\left(\frac{a}{2 M}\right)=\frac{1}{64} .
\end{aligned}
$$

So $\left(\mathrm{H}_{1}\right)$ is satisfied.

If $0 \leq u \leq 1600,-1600 \leq v \leq 0,0 \leq w, l \leq 1600$, then

$$
\begin{aligned}
f(u, v, w, l) & <10 \sqrt[10]{1600}+\frac{3}{1000}+\frac{40 \times 2}{1000} \\
& <(1-\beta) \phi_{p}\left(\frac{c}{2 M}\right)=32000 .
\end{aligned}
$$

So $\left(\mathrm{H}_{2}\right)$ is satisfied.

$$
\text { If } 1 \leq u \leq 1296,-1296 \leq v \leq 0,0 \leq w, l \leq 1296 \text {, then }
$$$$
f(u, v, w, l)>10 \sqrt[10]{1}+\frac{1}{1000}>\phi_{p}\left(\frac{b}{\delta L}\right)=\frac{3125}{648} .
$$

So $\left(\mathrm{H}_{3}\right)$ is satisfied.

For any $(u, v, w, l) \in[0,1600] \times[-1600,0] \times[0,1600] \times$ $[0,1600]$, we have

$$
\begin{gathered}
\min f(u, v, w, l) \geq \frac{1}{1000}, \\
\max f(u, v, w, l) \leq 10 \sqrt[10]{1600}+\frac{3}{1000}+\frac{40 \times 2}{1000}, \\
\phi_{p}\left(\frac{M}{2 m_{0}}\right)=2 \cdot 150^{2}, \quad \int_{0}^{1-\delta} q(t) d t=\frac{8}{5} \\
\int_{0}^{1} q(t) d t=\frac{1141}{625} .
\end{gathered}
$$

Hence, it's easy to know that $\left(\mathrm{H}_{4}\right)$ is satisfied.

So by Theorem 8 , we conclude that the BVP (21) has three positive solutions $u_{1}, u_{2}$, and $u_{3}$ satisfying

$$
\begin{gathered}
\left\|u_{1}\right\|<\frac{1}{2}, \quad 1<\min _{\delta \leq t<(1-\delta)} u_{2}(t), \quad\left\|u_{3}\right\|>\frac{1}{2} \\
\min _{\delta \leq t<(1-\delta)} u_{3}(t)<1 .
\end{gathered}
$$

\section{Acknowledgments}

This paper is supported by the Natural Science Foundation of China (10901045) and (11201112), the Natural Science Foundation of Hebei Province (A2009000664) and (A2011208012) and the Foundation of Hebei University of Science and Technology (XL200757).

\section{References}

[1] J. Wang, "The existence of positive solutions for the onedimensional p-Laplacian," Proceedings of the American Mathematical Society, vol. 125, no. 8, pp. 2275-2283, 1997.

[2] L. Kong and J. Wang, "Multiple positive solutions for the onedimensional p-Laplacian," Nonlinear Analysis, vol. 42, no. 8, pp. 1327-1333, 2000.

[3] X. He and W. Ge, "Twin positive solutions for the onedimensional $p$-Laplacian boundary value problems," Nonlinear Analysis, vol. 56, no. 7, pp. 975-984, 2004.

[4] D. Zhao, H. Wang, and W. Ge, "Existence of triple positive solutions to a class of $p$-Laplacian boundary value problems," Journal of Mathematical Analysis and Applications, vol. 328, no. 2, pp. 972-983, 2007.

[5] A. Lakmeche and A. Hammoudi, "Multiple positive solutions of the one-dimensional p-Laplacian," Journal of Mathematical Analysis and Applications, vol. 317, no. 1, pp. 43-49, 2006.

[6] D.-X. Ma, "Existence and iteration of positive solution for a three-point boundary value problem with a $p$-Laplacian operator," Journal of Applied Mathematics \& Computing, vol. 25, no. 1-2, pp. 329-337, 2007. 
[7] H. Feng and W. Ge, "Existence of three positive solutions for $m$-point boundary-value problems with one-dimensional $p$ Laplacian," Nonlinear Analysis, vol. 68, no. 7, pp. 2017-2026, 2008.

[8] Y. Guo, C. Yu, and J. Wang, "Existence of three positive solutions for $m$-point boundary value problems on infinite intervals," Nonlinear Analysis, vol. 71, no. 3-4, pp. 717-722, 2009.

[9] D. Kong, L. Liu, and Y. Wu, "Triple positive solutions of a boundary value problem for nonlinear singular secondorder differential equations of mixed type with $p$-Laplacian," Computers \& Mathematics with Applications, vol. 58, no. 7, pp. 1425-1432, 2009.

[10] J.-X. Hu and D.-X. Ma, “Triple positive solutions of a boundary value problem for second order three-point differential equations with $p$-Laplacian operator," Journal of Applied Mathematics and Computing, vol. 36, no. 1-2, pp. 251-261, 2011. 


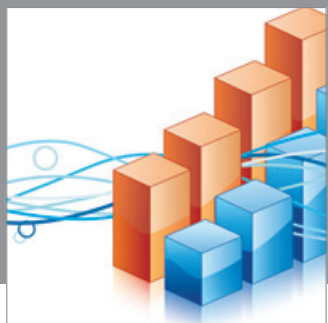

Advances in

Operations Research

mansans

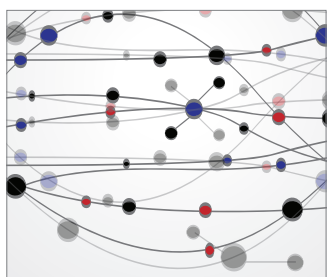

The Scientific World Journal
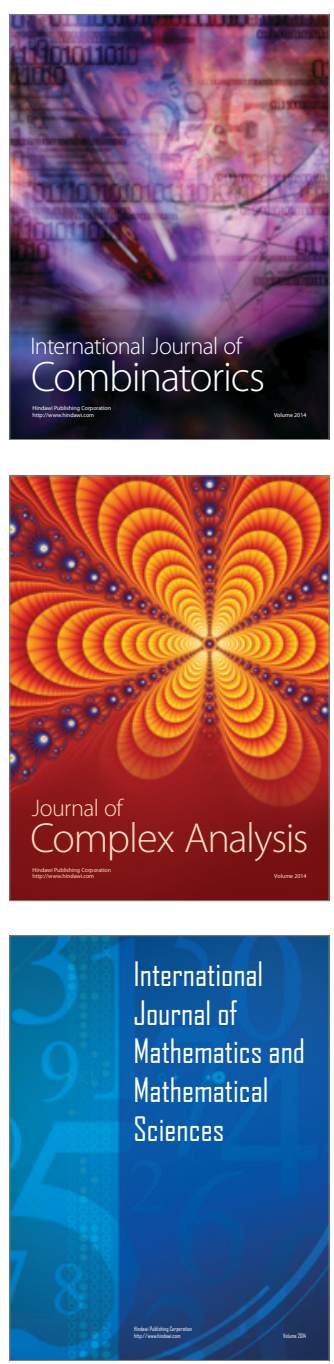
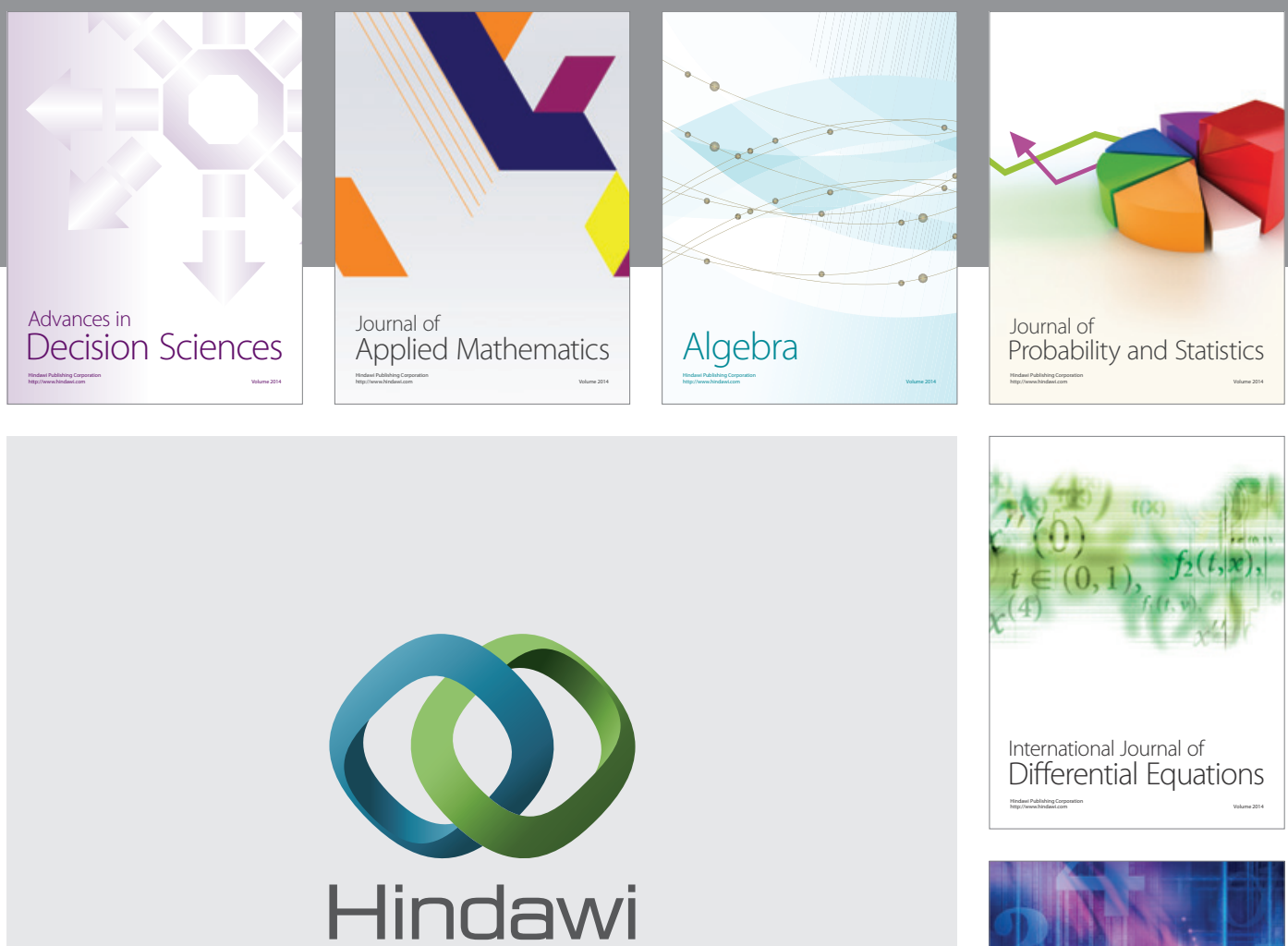

Submit your manuscripts at http://www.hindawi.com
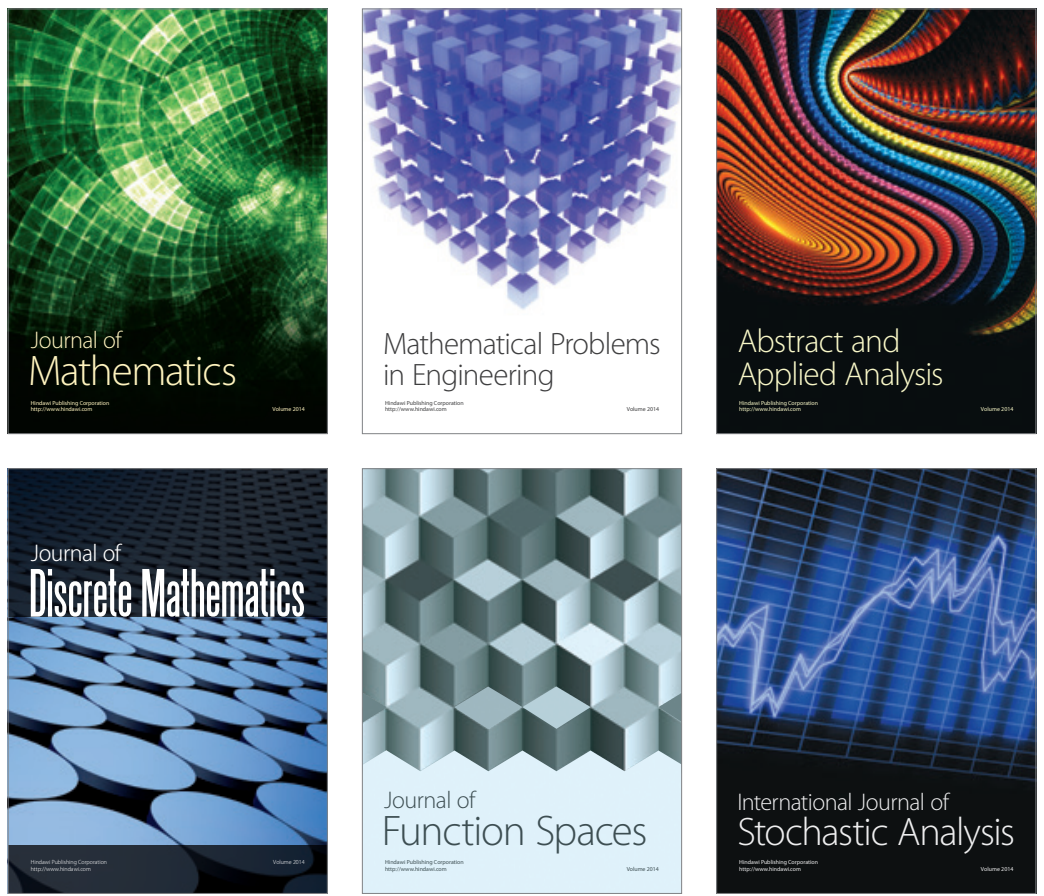

Journal of

Function Spaces

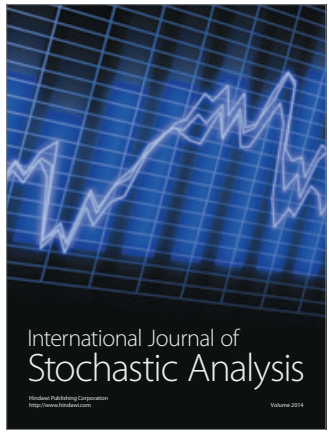

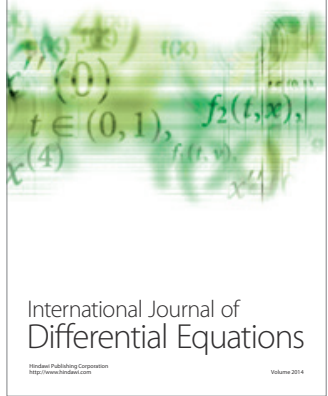
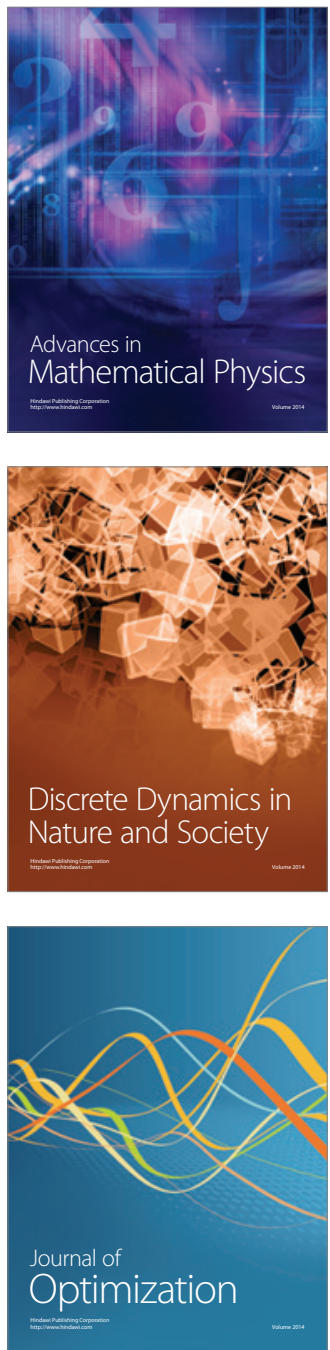